\title{
Better research needed for implants in grafted maxillary sinuses
}

Tong DC, Rioux K, Drangsholt M, Beirne OR. A Review of Survival Rates for Implants Placed in Grafted Maxillary Sinuses Using Meta-analysis. Int J Oral Maxillofac Implants 1998; 13: 175-181

Objectives To evaluate the survival of the implants placed into various materials used in the maxillary sinus lift procedure. The materials were autogenous bone hydroxyapatite (HA) alone or in combination with autogenous bone or demineralized freeze-dried bone (DFDB).

Data sources Medline computer search of the English literature between 1980 and 1996.

Study selection Only 10 of 28 identified studies met the inclusion criteria of $>10$ patients in each study all receiving root form endosseous implants, $<5 \%$ loss in follow-up over 6 months and reporting of survival data of the implants.

Data extraction and synthesis Proportion of surviving implants calculated with $95 \%$ confidence intervals. Patients were followed up for up to 60 months.

\begin{tabular}{|c|c|c|c|c|c|}
\hline Graft material & $\begin{array}{l}\text { No of } \\
\text { studies }\end{array}$ & $\begin{array}{c}\text { No of } \\
\text { implants }\end{array}$ & $\begin{array}{c}\text { No of } \\
\text { failures }\end{array}$ & $\begin{array}{c}\% \\
\text { survival }\end{array}$ & $\begin{array}{c}95 \% \\
\text { C.I. }\end{array}$ \\
\hline Autogenous bone & 6 & 484 & 47 & 90 & $87-93$ \\
\hline HA alone & 1 & 30 & 4 & 87 & $68-95$ \\
\hline HA autogenous bone & 3 & 363 & 22 & 94 & $90-97$ \\
\hline $\mathrm{HA} / \mathrm{DFDB}$ & 1 & 215 & 3 & 98 & $96-100$ \\
\hline
\end{tabular}

Conclusions These studies suggest that implant survival rates were similar for autogenous bone, HA/autogenous bone mix, HA/DFDB, and HA alone.

Address for reprints: Dr O Ross Beirne, Dept of Oral and Maxillofacial Surgery, School of Dentistry, University of Washington, Box 57134, Seattle, WA 98195-7134, USA.

\section{Commentary}

The authors begin by stating that studies reporting the short- and longterm outcomes of implant placement in the grafted maxillary sinus tend to be based on small numbers of subjects. They correctly imply that situation is one where meta-analysis can be usefully used to combine the datasets from individual studies to reveal more certain findings. The devil in meta-analysis comes in the process of data selection and combination; it is on this process that such exercises should be judged. In this case the authors undertake a comprehensive literature review yielding 28 studies which they then whittle down to 10 using reasonable criteria to define acceptable studies. Helpfully they report on the reasons for exclusion of the remainder. The authors used only the measure of implant survival expressed as the proportion surviving at the longest followup time in each particular study. It was not possible to calculate, for example, simple one-year survival rates because of differing lengths of follow-up and different reporting mechanisms. This is a great pity but the authors made the best of the disparate data available. They report in some detail the differences between the studies that limited this process. This report also reminded the reviewer of how little consistency currently exists in terms of surgical technique and reporting of data in this interesting area of dental care. The inconsistent follow-up methods of many of the studies was not excusable, however, and future research in this area would benefit from some sensible protocol development.

Overall the authors report a mean survival rate of between $87 \%$ and $98 \%$ depending on the type of bone augmentation utilised. The best retention rate was seen when a mixture of freezedried bone and hydroxyapatite was used, whereas autogenous bone alone was the least useful. These findings, however, require a great deal of caution in interpretation as many of the techniques were based on only a single paper with very small numbers included. Overall the authors sensibly comment that about the only conclusion that can be drawn is that all techniques seem at least moderately successful and all have retention rates of the same magnitude.

The authors have made a reasonable attempt to undertake a meta-analysis of this area but, as they report themselves, the validity of this process was strongly limited by the very poor data available. Perhaps the most useful finding of this paper is that there is an urgent need for a well-controlled multicentre trial of this technique.

\section{Mark Brickley}

Dept of Oral and Maxillofacial Surgery, Welsh National School of Medicine, UK 